\title{
Numerical approximation of a two-dimensional nonlinear and non-equilibrium model of reactive chromatography
}

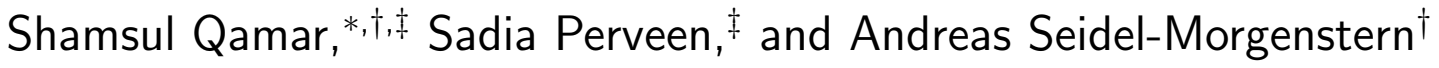 \\ †*Max Planck Institute for Dynamics of Complex Technical Systems, Magdeburg, Germany \\ $\ddagger$ Department of Mathematics, COMSATS Institute of Information Technology, Islamabad, \\ Pakistan \\ E-mail: *qamar@mpi-magdeburg.mpg.de
}

\begin{abstract}
A two-dimensional nonlinear and non-equilibrium model of liquid chromatography is numerically approximated to simulate the dynamics of multi-component mixtures in a fixed-bed isothermal liquid chromatographic reactor. The mathematical model is formed by a system of nonlinear convection-diffusion-reaction partial differential equations coupled with differential and algebraic equations. A semi-discrete high resolution finite volume scheme is applied to solve the model equations. The scheme is second order accurate in axial and radial coordinates. The resulting system of ordinary differential equations is solved by a second order accurate Runge-Kutta method. The proposed scheme capably captures narrow peaks and sharp discontinuities in the concentration profiles. Radial gradients were not considered in the pervious studies which are particularly important in the case of non-perfect injections. Several test problems of heterogeneously catalyzed reversible reactions are carried out. The considered case studies include three and four-component elution assuming hypothetical injections of
\end{abstract}


the reactants in inner or outer sections of the column inlet cross-section. The developed numerical algorithm and results are useful tools for further improvements in reactive chromatography.

\section{Introduction}

Reactive chromatography, is an attractive separation technique for chemical synthesis. It combines chemical reactions and chromatographic separations into a single unit for the production of high purity products. The technique is successfully employed to significantly improve the process performance of many multi-functional reactors. It reduces capital investment, equipment size, cost, energy, pollution and waste, while improves productivity and purity. $^{1-20}$

Chromatographic reactors separate reactants and products on the basis of differences in the selective adsorption of different components. Within a chromatographic reactor, the conversion of reactants and the separation of components take place simultaneously. In order to understand the basic concept of a batch reactor, let us consider a reversible reaction of the type $\mathrm{C} \leftrightarrows \mathrm{A}+\mathrm{B}$ in a single chromatographic column (c.f. Figure 1). In this case, rectangular pulses of the reactant $\mathrm{C}$ are periodically injected into the column packed with a stationary phase. The reaction occurs at the surface of the catalyst to form the products B and C. As the reaction is taking place, the components interact with the surface of adsorbent. Different affinities of the components $\mathrm{A}, \mathrm{B}$ and $\mathrm{C}$ to the stationary phase produce different migration velocities of these components, leading to their separations. The process enhances the driving force for the forward reaction, suppresses the backward reaction, overcomes the chemical equilibrium, and provides high conversion of the reactant $\mathrm{C}$ at the column outlet. Complete conversion could be possible if the residence time of the reactant $\mathrm{C}$ in the column is long enough.

The models of isothermal reactive chromatography can be described by a coupled nonlinear system of convection-diffusion-reaction type partial differential equations (PDEs) and alge- 
braic or differential equations describing the kinetic phenomena. ${ }^{1,21,22}$ Due to the nonlinearity of model equations, numerical solution techniques are required to get accurate solutions. ${ }^{22-25}$ Modeling and numerical simulation of the production processes have gained immense industrial popularity during the recent years. Numerical solution techniques have a distinctive advantage over traditional empirical method, as they enable us to understand the physical meaning of these models. Numerical techniques are helpful in analyzing the response of a system to different sets of parameters and to optimize several factors, such as productivity, purity, production rate, and eluent consumption. ${ }^{22}$

The finite volume schemes have been widely applied to simulate different 1D chromatographic models and were found to be a good choice for the numerical simulation of such nonlinear convection dominated problems. ${ }^{23,26-28}$ These schemes were initially introduced for nonlinear hyperbolic equations and are based on three main features namely, formal integration, discretization and solution. Such type of schemes are stable and high order accurate on coarse grids. The schemes resolve sharp variations by avoiding numerical oscillations and over-predictions in the solutions. ${ }^{24,25,29}$

This study extends and generalizes our recent study for linear two-dimensional reactive lumped kinetic model (2D-RLKM) to nonlinear 2D-RLKM. ${ }^{30}$ Injections of specific profiles are considered to amplify the effect of possible rate limitations of the mass transfer in the radial direction. The high resolution finite volume scheme (HR-FVS) of Koren is extended to solve the current $2 \mathrm{D}$ model equations. ${ }^{23,29}$ The scheme is robust and second order accurate in axial and radial-coordinates. It has capability to resolves sharp discontinuities of the elution profiles and avoids numerical dispersion. Several challenging case studies of threeand four-component elution are carried out. The numerical results are critically evaluated to elucidate the effects of different kinetic parameters on conversion and separation.

The current $2 \mathrm{D}$ reactive model can be useful in various situations namely, i) the injection at the column inlet is not perfect (i.e. a radial profile is introduced at the column inlet), ii) the column is not homogeneously packed (which is more probable in the case of larger columns), 
iii) there are radial temperature gradients, which are connected also with radial concentration gradients. All these scenarios occur in reality. Often they are minor or even negligible, then 1D models are applicable. However, for their relevance and effects the investigation 2D models are necessary. With our current isothermal nonlinear-reactive model we are able to study situation i) only by assuming injections in inner cylinders or outer annuli. Situations ii) and iii) are more complicated and need further model extensions (considering non-constant column porosities and an energy balance), which are currently under investigation. Thus, the develop numerical algorithm and results are helpful tools to further develop 2D nonlinear chromatographic reactors. These results could be used to study the effects of mass transfer kinetics, reaction kinetics, and axial and radial dispersion coefficients on the elution profiles. The studied 2D-model and numerical scheme are more general and flexible than the classical 1D-models and numerical schemes. ${ }^{14-16,31}$ Further, we have provided useful numerical tools to approximate and apply this model, if required. The latter means if radial dispersion is rate limiting.

The rest of the paper is organized as follows. In Section 2, the 2D-RLKM model is briefly introduced. In Section 3, the high resolution finite volume scheme is derived. In Section 4, different case studies are carried out. Finally, conclusions are drawn in Section 5.

\section{Nonlinear 2D-RLKM}

In the case of multi-component nonlinear 2D-RLKM, the mass balances for concentrations in the liquid phase are coupled with the differential kinetic equations for solid phase concentrations. The model assumes that the kinetics of adsorption-desorption are infinitely fast but the mass transfer kinetics are not. The model lumps the contribution of internal and external mass transport resistances into a mass transfer coefficient $k$. In this study, heterogeneously catalyzed solid phase reversible reactions are considered. The reactants and products travel along the column axis in the $z$-direction by advection and axial dispersion, 
spread along the column radius in the $r$-direction by radial dispersion, and reactants are continuously decaying into produce products due to chemical reactions in the solid phase. The flow rate variations are neglected and the interstitial velocity $u$ is kept constant. The following particular injection conditions are assumed to intensify the effects of mass transfer rate in the radial-direction. The inlet cross sectional area of the cylindrical column is divided into two regions by introducing a new parameter $\tilde{r}$, referred as inner-cylindrical and outer-annular regions (see Figure 1). There are three possibilities for the reactant injection namely, an inner region injection, an outer region injection, and injection through the whole cross-section. The injection over the whole cross-section is possible when the radius of the column, denoted by $R$, is set equal to the inner zone radius $\tilde{r}$. We must point out that the case of injecting the sample through the outer annular ring has some similarity with process of annular chromatography. ${ }^{32}$ However, during annular chromatography the column rotates. Under these assumptions, the mass balance equations for concentrations of mixture components in the liquid phase can be expressed as ${ }^{21,22,30}$

$$
\frac{\partial c_{i}}{\partial t}+u \frac{\partial c_{i}}{\partial z}=D_{z, i} \frac{\partial^{2} c_{i}}{\partial z^{2}}+D_{r, i} \frac{1}{r} \frac{\partial}{\partial r}\left(r \frac{\partial c_{i}}{\partial r}\right)-\frac{k_{i}}{\epsilon}\left[q_{i}^{*}-q_{i}\right]
$$

The corresponding mass balances for mixture components concentrations in the stationary phase accounting for solid phase reversible reaction can be expressed $\operatorname{as}^{21,22,30}$

$$
\frac{\partial q_{i}}{\partial t}=\frac{k_{i}}{(1-\epsilon)}\left[q_{i}^{*}-q_{i}\right]+\sum_{n=1}^{N_{R}} \nu_{i, n} r_{n}^{\text {het }}, \quad i=1,2, \cdots, N_{c}
$$

In the above equations $c_{i}(t, z, r)$ and $q_{i}(t, z, r)$ denote the $i$-th component solute concentrations in the liquid and solid phases, respectively, $r_{n}^{\text {het }}$ is reaction rate in the solid phase (heterogeneous) and $\nu_{i, n}$ are stoichiometric coefficients of component $i$ in reaction $n$, generally, $\nu_{i, n}=-1$ for reactants and $\nu_{i, n}=+1$ for products. Moreover, $D_{z, i}$ and $D_{r, i}$ represent the longitudinal and radial dispersion coefficients of $i$-th component, $\epsilon \in(0,1)$ is the external porosity, $N_{R}$ is the number of reactions involved and $N_{c}$ denotes the number of mixture 
components. In addition, $t, z$ and $r$ denote the time, axial and radial coordinates, respectively. For sufficiently large values of $k_{i}$, for $i=1,2, \ldots N_{c}$, the solution of multi-component 2D-RLKM converges to that of multi-component $2 \mathrm{D}$ reactive equilibrium dispersive model (2D-REDM).

For modeling and simulation of preparative chromatography, experimentally determined adsorption equilibrium data has to be represented by suitable mathematical equations. Different relations of isotherms are available in the literature. ${ }^{21,22}$ Here, we consider the commonly used nonlinear convex Langmuir isotherm ${ }^{22}$

$$
q_{i}=\frac{a_{i} c_{i}}{1+\sum_{n=1}^{N_{c}} b_{n} c_{n}}, \quad i=1,2,3, \ldots . . N_{c}
$$

where $a_{i}$ represents the Henry's coefficient of $i$-th component and $b_{n}$ quantifies the nonlinearity of the isotherm. To simply the notations and reduce the number of variables appearing in the model equations, the following dimensionless quantities are introduced:

$$
x=\frac{z}{L}, \quad \tau=\frac{u t}{L}, \quad \rho=\frac{r}{R}, \quad P e_{z, i}=\frac{L u}{D_{z, i}}, \quad P e_{r, i}=\frac{R^{2} u}{D_{r, i} L} \quad \kappa_{i}=\frac{k_{i} L}{u},
$$

where $L$ is the height of the column, $P e_{z, i}$ and $P e_{r, i}$ are the dimensionless Peclet numbers in longitudinal and radial-directions, respectively. Using the above dimensionless variables, the model equations in Eqs. (1) and (2) can be rewritten as

$$
\begin{gathered}
\frac{\partial c_{i}}{\partial \tau}=\frac{1}{P e_{z, i}} \frac{\partial^{2} c_{i}}{\partial x^{2}}-\frac{\partial c_{i}}{\partial x}+\frac{1}{P e_{r, i}} \frac{1}{\rho} \frac{\partial}{\partial \rho}\left(\rho \frac{\partial c_{i}}{\partial \rho}\right)-\frac{\kappa_{i}}{\epsilon}\left(q_{i}^{*}-q_{i}\right), \\
\frac{\partial q_{i}}{\partial \tau}=\frac{\kappa_{i}}{(1-\epsilon)}\left(q_{i}^{*}-q_{i}\right)+\frac{L}{u} \sum_{n=1}^{N_{R}} \nu_{i, n} r_{n}^{\text {het }} .
\end{gathered}
$$

Here, $0 \leq \rho \leq 1$ and $0 \leq x \leq 1$. The model equations represented by Eqs. (5)-(6) are 
also subjected to the appropriate initial conditions, as well as inlet and outlet boundary conditions. The initial conditions for an initially equilibrated column are given as

$$
c_{i}(\tau=0, x, \rho)=c_{i, \text { init }}, \quad q_{i}(\tau=0, x, \rho)=q_{i, \text { init }}^{*}, \quad i=1,2,3, \ldots \ldots N_{c} .
$$

The radial boundary conditions $(\mathrm{BCs})$ at $\rho=0$ and $\rho=1$ are expressed as ${ }^{30,34}$

$$
\frac{\partial c_{i}(\tau, x, \rho=0)}{\partial \rho}=0, \quad \frac{\partial c_{i}(\tau, x, \rho=1)}{\partial \rho}=0
$$

In this study, the Danckwert's BCs are considered at the column inlet. ${ }^{33}$ For the inner zone injection, these BCs are expressed as ${ }^{30,34}$

$$
c_{i}(\rho, x=0, \tau)-\frac{1}{P e_{z, i}} \frac{\partial c_{i}(\rho, x=0, \tau)}{\partial x}= \begin{cases}c_{i, \mathrm{inj}}, & \text { if } 0 \leq \rho \leq \tilde{\rho} \text { and } 0 \leq \tau \leq \tau_{\text {inj }} \\ 0, & \tilde{\rho}<\rho \leq 1 \text { or } \tau>\tau_{\text {inj }}\end{cases}
$$

while, for the injection through outer annular zone they are given as

$$
c_{i}(\rho, x=0, \tau)-\frac{1}{P e_{z, i}} \frac{\partial c_{i}(\rho, x=0, \tau)}{\partial x}= \begin{cases}c_{i, \text { inj }}, & \text { if } \tilde{\rho}<\rho \leq 1 \text { and } 0 \leq \tau \leq \tau_{\text {inj }} \\ 0, & 0 \leq \rho \leq \tilde{\rho} \text { or } \tau>\tau_{\text {inj }}\end{cases}
$$

together with the Neumann condition at the outlet of a finite length column

$$
\left.\frac{\partial c_{i}}{\partial x}\right|_{x=1}=0
$$

The symbol $c_{i, \text { inj }}$ represents the concentration of injected $i$-th component, $\tau_{\text {inj }}$ denotes the dimensionless time of injection, and

$$
\tilde{\rho}=\tilde{r} / R
$$

For injection over the whole inlet cross-section of the column, either $\tilde{\rho}=1$ in Eq. (9) or 
$\tilde{\rho}=0$ in Eq. (10).

At the column outlet, the following Neumann BCs are used:

$$
\frac{\partial c_{i}(\tau, x=1, \rho)}{\partial x}=0
$$

\section{Numerical Schemes}

Various numerical procedures have been introduced in the literature for approximating the one-dimensional chromatographic models. ${ }^{22,23}$ In this work, the $1 \mathrm{D}$ semi-discrete high resolution flux-limiting finite volume scheme (HR-FVS) of Koren is extended to solve the current two-dimensional system of PDEs. ${ }^{23,29}$ The scheme is second order accurate in the axial and radial-coordinates. The resulting system of ordinary differential equations (ODEs) is solved by using a second-order Runge-Kutta method. Here, the HR-FVS is applied to the PDEs in Eq. (5) only, while ODEs in Eq. (6) are solved by the considered ODE-solver.

Before applying the proposed numerical scheme to Eq. (5), the first step is to discretize the computational domain. Let $N_{x}$ and $N_{\rho}$ be the large integers in $x$ and $\rho$-directions, respectively. We assume a Cartesian grid with a rectangular domain $[0,1] \times[0,1]$ which is

covered by cells $\Omega_{j l} \equiv\left[x_{j-\frac{1}{2}}, x_{j+\frac{1}{2}}\right] \times\left[\rho_{l-\frac{1}{2}}, \rho_{l+\frac{1}{2}}\right]$ for $1 \leq j \leq N_{x}$ and $1 \leq l \leq N_{\rho}$. The representative coordinates in the cell $\Omega_{j l}$ are denoted by $\left(x_{j}, \rho_{l}\right)$. Here

$$
\left(x_{1 / 2}, \rho_{1 / 2}\right)=(0,0), \quad x_{j}=\frac{x_{j-1 / 2}+x_{j+1 / 2}}{2}, \quad \rho_{l}=\frac{\rho_{l-1 / 2}+\rho_{l+1 / 2}}{2}
$$

and

$$
\Delta x_{j}=x_{j+1 / 2}-x_{j-1 / 2}, \quad \Delta \rho_{l}=\rho_{l+1 / 2}-\rho_{l-1 / 2} .
$$


The cell averaged values of $w_{i, j, l}(\tau)$ at any time $\tau$ are given as

$$
w_{i, j, l}=w_{i, j, l}(\tau)=\frac{1}{\Delta x_{j} \Delta \rho_{l}} \int_{\Omega_{j l}} w_{i}(\tau, x, \rho) d x d \rho
$$

Here, $w \in\left\{c, q, q^{*}\right\}$. Till now, the computational domain is discretized and the corresponding initial data for $\tau=0$ are allocated to each mesh interval.

Now, integration of Eqs. (5) and (6) over $\Omega_{j l}$ give

$$
\begin{gathered}
\frac{d c_{i, j, l}}{d \tau}=-\frac{c_{i, j+\frac{1}{2}, l}-c_{i, j-\frac{1}{2}, l}}{\Delta x_{j}}+\frac{1}{\Delta x_{j} P e_{z}}\left[\left(\frac{\partial c}{\partial x}\right)_{i, j+\frac{1}{2}, l}-\left(\frac{\partial c}{\partial x}\right)_{i, j-\frac{1}{2}, l}\right] \\
+\frac{1}{\Delta \rho_{l} P e_{r} \rho_{l}}\left[\left(\rho \frac{\partial c}{\partial \rho}\right)_{i, j, l+\frac{1}{2}}-\left(\rho \frac{\partial c}{\partial \rho}\right)_{i, j, l-\frac{1}{2}}\right]-\frac{\kappa}{\epsilon}\left(q_{i, j, l}^{*}-q_{i, j, l}\right), \\
\frac{d q_{i, j, l}}{d \tau}=\frac{\kappa}{1-\epsilon}\left(q_{i, j, l}^{*}-q_{i, j, l}\right)+\sum_{n=1}^{N_{R}} \nu_{i, n} r_{n, j, l}^{\text {het }} .
\end{gathered}
$$

Different approximations of the cell interface concentrations along axial-coordinate generate different numerical schemes which are discussed below.

First-order scheme: In this case, the values of concentrations at the cell interfaces in Eq. (17) are approximated as

$$
c_{i, j+\frac{1}{2}, l}=c_{i, j, l}, \quad c_{i, j-\frac{1}{2}, l}=c_{i, j-1, l}
$$

This approximation yields a first-order accurate scheme in the axial-direction. The above approximations provide a first order accurate scheme in the axial and radial coordinates.

HR-FVS of Koren: The following flux-limiting formula is applied to approximate the cell interface values $c_{i, j, l+\frac{1}{2}}$ of the concentration in Eq. (17): ${ }^{29}$

$$
c_{i, j+\frac{1}{2}, l}=c_{i, j, l}+\frac{1}{2} \psi\left(\theta_{i, j+\frac{1}{2}, l}\right)\left(c_{i, j, l}-c_{i, j-1, l}\right),
$$


where,

$$
\theta_{i, j+\frac{1}{2}, l}=\frac{c_{i, j+1, l}-c_{i, j, l}+\beta}{c_{i, j, l}-c_{i, j-1, l}+\beta}
$$

is the ratio of concentration gradients. Here, division by zero can be avoided by considering $\beta=10^{-10}$. The limiting function $\psi$ is taken $\operatorname{as}^{29}$

$$
\psi\left(\theta_{i, j+\frac{1}{2}, l}\right)=\max \left(0, \min \left(2 \theta_{i, j+\frac{1}{2}, l}, \min \left(\frac{1}{3}+\frac{2}{3} \theta_{i, j+\frac{1}{2}, l}, 2\right)\right)\right)
$$

Similarly, $c_{i, j-\frac{1}{2}, l}$ can be calculated by replacing the index $j$ by $j-1$ in the above equations. Other flux-limiting schemes: Several other flux-limiting schemes are available in the literature. These schemes are different because each scheme involve different flux-limiting functions. ${ }^{24,35}$ In these schemes, a limited right cell boundary flux, $\Omega_{j, l}$ is defined as

$$
c_{i, j+\frac{1}{2}, l}=c_{j, l}+\frac{1}{2} \varphi\left(\eta_{i, j+\frac{1}{2}, l}\right)\left(c_{i, j+1, l}-c_{i, j, l}\right)
$$

The left cell-boundary flux can be approximated in an analogous manner. Here, $\eta_{j+\frac{1}{2}, l}$ is given by the following ratio

$$
\eta_{i, j+\frac{1}{2}, l}=\frac{c_{i, j, l}-c_{i, j-1, l}+\beta}{c_{i, j+1, l}-c_{i, j, l}+\beta}
$$

A few well known flux limiters are listed in Table 1.

Approximations of axial and radial differential terms: The differential values of the concentration at the cell interfaces obtained from the approximation of the axial dispersion term in Eq. (17) are calculated as

$$
\left(\frac{\partial c}{\partial x}\right)_{i, j \pm \frac{1}{2}, l}= \pm\left(\frac{c_{i, j \pm 1, l}-c_{i, j, l}}{\Delta x_{j}}\right)
$$

Moreover, the differential cell interface values in the radial dispersion term of Eq. (17) are 
calculated as

$$
\left(\rho \frac{\partial c}{\partial \rho}\right)_{i, j, l+\frac{1}{2}}=\rho_{l} \max \left(\frac{c_{i, j, l+1}-c_{i, j, l}}{\Delta \rho_{l}}, 0\right)+\rho_{l+1} \min \left(\frac{c_{i, j, l+1}-c_{i, j, l}}{\Delta \rho_{l}}, 0\right)
$$

Similarly, the left interface values can be calculated by lowering the index $l$ by one.

Scheme strategy at the boundaries: The flux-limiting formula given by Eq. (20) is not applicable to the boundary cells. In that case, the first order scheme is applied to approximate the cell interface values of the concentration in the boundary cells. In the remaining interior cells, one of the above mentioned second order accurate HR-FVS schemes can be applied.

ODE-solver: To obtain the second order accuracy in time, a second order total variation

diminishing (TVD) Runge-Kutta scheme is applied to solve Eqs. (17) and (18). ${ }^{36}$ Denoting the right-hand side of Eqs. (17) and (18) as $\mathrm{E}(w)$ for $w \in\{c, q\}$, a second order TVD Runge-Kutta scheme update $w$ through the following two stages

$$
\begin{aligned}
w^{(1)} & =w^{n}+\Delta \tau \mathrm{E}\left(w^{n}\right), \\
w^{n+1} & =\frac{1}{2}\left(w^{n}+w^{(1)}+\Delta \tau \mathrm{E}\left(w^{(1)}\right)\right),
\end{aligned}
$$

where $w^{n}$ is a solution at previous time step $\tau^{n}$ and $w^{n+1}$ is updated solution at next time step $\tau^{n+1}$. Moreover, $\Delta \tau$ represents the time step which is calculated under the following Courant-Friedrichs-Lewy (CFL) condition

$$
\Delta \tau \leq 0.5 \min \left(\Delta x, \Delta x^{2} P e_{z}, \Delta \rho^{2} P e_{r}, \frac{1-\epsilon}{\kappa}\right)
$$

\section{Numerical case studies}

In this section several test problems are considered. The case studies include practical examples of three- and four-component elution considering nonlinear heterogeneous reactions. The parameters used in these test problems are taken from Tien ${ }^{37}$ which have been chosen in 
accordance with ranges typically encountered in HPLC applications. ${ }^{22}$ The axial and radial dispersion coefficients are taken the same for all mixture components, i.e. $D_{z, i}=D_{z}$ and $D_{r, i}=D_{r}$, for $i=1,2, \cdots, N_{c}$. Moreover, the mass transfer coefficients are also taken the same for all components, i.e. $k_{i}=k$. However, the current model equations and the proposed numerical solution technique allow considering different mass transfer coefficients for each specie. Thus, different mass transfer coefficients can also be considered for each component when required.

In the numerical case studies, we tried different combinations of grid points. It was found that the scheme gives good resolution of profiles on $100 \times 50$ grid points. Thus, we kept the number of gird points fixed to $100 \times 50$.

\section{Error analysis for single-component non-reactive elution}

The purpose of this simple case study is to verify the accuracy and efficiency of the proposed HR-FVS of Koren against other other flux-limiting HR-FVS given above. Here, radial effects are neglected by considering a large radial dispersion coefficient, i.e. $D_{r}=0.5$. Thus, the solution of current 2D-problem is equivalent to the solution of $1 \mathrm{D}$-problem. ${ }^{14}$ It is further assumed that the column is partially pre-loaded in the region $[0.2,0.4] \times[0,1]$ by a sinusoidal profile. The initial conditions are taken as

$$
c(0, x, \rho)= \begin{cases}\sin (\pi(x-0.2) / 0.2), & 0.2 \leq x \leq 0.4 \& 0 \leq \rho \leq 1 \\ 0, & \text { otherwise }\end{cases}
$$

Nothing is injected at the column inlet, i.e. $c(t, 0, \rho)=0$, while the zero Neumann BCs are considered at the outlet of the column. Moreover, $L=1.0 \mathrm{~cm}, R=0.1 \mathrm{~cm}, a=1$, $u=1 \mathrm{~cm} / \mathrm{min}, \epsilon=0.5$, and $k=50 \mathrm{~min}^{-1}$, respectively. The simulation time is taken to be $0.6 \mathrm{~min}$. The $L^{1}$-error along the axial-coordinate of the column at $\rho=0$ and at 
$t_{\max }=0.6 \mathrm{~min}$ is obtained by considering the following formula

$$
L^{1} \text {-error }=\sum_{j=1}^{N_{x}}\left|c_{\text {exact }}\left(t=0.6, x_{j}, \rho=0\right)-c_{\text {Numeric }}\left(t=0.6, x_{j}, \rho=0\right)\right| \Delta x
$$

where $c_{\text {exact }}$ represents the exact solution and $c_{\text {Numeric }}$ denotes the corresponding numerical solution. Moreover, $N_{x}$ denotes the number of discretization points in the axial-coordinate and $\Delta x$ represents the axial step-size.

Due to neglected radial effects, the solution has the same value at all discrete points of the radial-coordinate. Further, $k$ is assumed very large. Thus, the numerical solution obtained is compared with the following 1D analytical solution given by Koren ${ }^{29}$ using the same initial and boundary conditions:

$$
c_{\text {exact }}(t, z, \rho=0)=0.5 \operatorname{real}\left(i e^{p}[\operatorname{erf}(\alpha)-\operatorname{erf}(\beta)]\right),
$$

where, erf represents the error function and

$$
\begin{aligned}
& p=-0.5 D_{\mathrm{z}} t\left(\frac{\pi}{0.2}\right)^{2}+i \frac{\pi}{0.2}(0.2-z-0.5 t), \\
& \alpha=\frac{-0.2+z-0.5 t}{2 \sqrt{0.5 D_{\mathrm{z}} t}}-i \pi \frac{\sqrt{0.5 D_{\mathrm{z}} t}}{0.2} \\
& \beta=\frac{-0.4+z-0.5 t}{2 \sqrt{0.5 D_{\mathrm{z}} t}}-i \pi \frac{\sqrt{0.5 D_{\mathrm{z}} t}}{0.2} .
\end{aligned}
$$

Table 2 gives a comparison of $L^{1}$-errors of different numerical schemes. The analysis is performed for different values of axial-dispersion coefficients $D_{z}$ using $N_{x} \times N_{r}=100 \times 50$ grid points. It can be observed that the Koren scheme gives less errors as compared to the other flux-limiting schemes and has less computational cost. Table 3 displays the $L^{1}$-error and the experimental order of convergence (EOC) of the Koren scheme at different mesh points and for different values of $D_{z}$. The EOC of the Koren method is about second order (see Table 3) and, hence, seems more useful for such models. The computational cost for the 
Minmod limiter is higher than the other flux-limiting schemes. The backward difference (first order) scheme has minimum computational cost but produces large errors in the solution. The computational cost of the van-Leer limiter is comparable to the Koren scheme but it has large errors in the solutions. The remaining two limiters have a higher computational costs and lower accuracy. With these observations we can conclude that Koren scheme is a better choice for solving such models.

\section{Three-component reactive elution}

In this section, the proposed numerical scheme is applied on the model equations to simulate three-component reactive elution, i.e. $N_{c}=3$ and $N_{R}=1$. Two different case studies for the nonlinear reaction of type $C \leftrightarrows A+B$ are presented considering either linear or nonlinear adsorption isotherms. In both cases, the reactant is injected through inner cylindrical core of the column inlet cross-section. The parameters used in this test problem are taken from Tien $^{37}$ who also investigated this problem for the 1D-model.

\section{Case 1a: Reaction of type $C \leftrightarrows A+B$ with linear adsorption isotherm}

In this type of reaction, only component 3 (component $\mathrm{C}$ ), representing the reactant, is injected to the initially empty column, while component 1 (component A) and component 2 (component B) denote the reaction products. During its propagation though the column, the reactant $\mathrm{C}$ is continuously converting into the products $\mathrm{A}$ and $\mathrm{B}$ due to the cataltic nature of the solid bed. At the same time, all three components A, B and C are also separating due to their different affinities to the solid bed. The component having more interaction with the solid bed slows down as compared to the component having less interaction. Thus, at the end of the column separated concentration pulses of each component can be collected. The model Eqs. (5) and (6) with $N_{c}=3, N_{R}=1$, and linear equilibrium adsorption isotherms $q_{i}^{*}=a_{i} c_{i}$ for $i=1,2,3$ are numerically approximated. Only the solid phase (heterogeneous) reaction with reaction rate $r^{\text {het }}$ is considered, while the homogeneous liquid phase reaction 
is neglected, i.e. $r^{\text {hom }}=0$. The heterogeneous reaction rate is taken as

$$
r^{\text {het }}=k^{\text {het }}\left(q_{3}-\frac{q_{1} q_{2}}{k_{\mathrm{eq}}^{\mathrm{het}}}\right) .
$$

Here, $q_{1}, q_{2}$ and $q_{3}$, represent the solid phase concentrations of the products and reactant. Moreover, $k^{\text {het }}$ is the reaction rate constant and $k_{\text {eq }}^{\text {het }}$ is the chemical equilibrium constant. The signs of stoichiometric coefficients $\nu_{1}$ and $\nu_{2}$ for the products are taken positive, while the sign of $\nu_{3}$ for the reactant is taken negative.

The parameters used in this test problems are as follows. ${ }^{37}$ The column length is $L=25 \mathrm{~cm}$, column radius $R=1.25 \mathrm{~cm}$, radius of inner zone $\bar{r}=0.88375 \mathrm{~cm}$, mass transfer coefficient $k=50 \mathrm{~min}^{-1}$, dispersion coefficient $D_{z}=10^{-5} \mathrm{~cm}^{2} / \mathrm{min}, D_{r}=0.1 \mathrm{~cm}^{2} / \mathrm{min}$, porosity $\epsilon=0.25, k^{\text {het }}=5000 \mathrm{~min}^{-1}, k_{\text {eq }}^{\text {het }}=0.833 \mathrm{~mol} / l$, and adsorption coefficients are $a_{1}=5$, $a_{2}=1$ and $a_{3}=3$. Moreover, only component 3 with concentration $c_{3, \text { inj }}=0.5 \mathrm{~mol} / \mathrm{l}$ is injected to the reactor through inner cylindrical core for $t_{\text {inj }}=10 \mathrm{~min}$ with fluid velocity $u=100 \mathrm{~cm} / \mathrm{min}$. The $1 \mathrm{D}$ and $3 \mathrm{D}$ plots of the concentration profiles for $c_{1}, c_{2}$ and $c_{3}$ are generated using $100 \times 50$ grid points as shown in Figure 2. It can be seen that the Koren scheme resolves the discontinuous profiles of concentrations. It can also be observed in Figure 2 that reactant (component $\mathrm{C}$ ) is moving between the products (components $\mathrm{A}$ and B). This is a very favorable case which diminishes the backward reaction. The effect of radial dispersion is clearly visible in the 3D plots along $\tau$ and $\rho$ coordinates. It can be seen that concentration of the reactant (component 3) is decreasing and concentrations of the products (components 1 and 2) are increasing. Conversion of the reactant can be calculated as

$$
X_{C}[\%]=100 \times \frac{n_{C}^{\text {in }}-n_{C}^{\text {out }}}{n_{C}^{\text {in }}},
$$

where, $n_{C}^{\text {in }}$ represents the number of moles of component $C$ injected at the column inlet and $n_{C}^{\text {out }}$ denotes the number of moles at the column outlet. Figure 3 gives the plot of conversion with respect to the column length. The figure shows that complete conversion 
of the reactant could be possible if longer column is considered, i.e. if residence time of the reactant in the column is increased. However, there exist some other parameters (or variables) which could also be considered to increase the conversion, such as bed diameter, porosity, inlet concentrations, and etc.

The effects of $P e_{r}$ and mass transfer coefficient $k$ on component 1 are shown in Figure 4. It can be observed from the Figure 4a that the radial effects are more prominent for large values of $P e_{r}$ (or small $D_{r}$ ). For $P e_{r}=250$ the results of 2D-RLKM reduces to the results of 1D-RLKM. Figure 4b shows that the profile of component 1 is diffusive for small values of $k$ and becomes sharper on increasing the value of $k$. It was further observed that the effect of $P e_{z}$ on the elution profiles is similar to that of $k$ and its plot is therefore omitted.

\section{Case 1b: Reaction of type $C \leftrightarrows A+B$ with nonlinear isotherm}

Here, we extend the previous case of linear isotherm to the case of nonlinear adsorption isotherms for studying the effect of nonlinearity on the conversion of reactant and separation of products. The Langmuir isotherms given by Eq. (3) are considered. Two pairs of isotherm parameters are considered, such as (i) $b_{1}=1.0 \mathrm{l} / \mathrm{mol}, b_{2}=1.0 \mathrm{l} / \mathrm{mol}, b_{3}=1.0 \mathrm{l} / \mathrm{mol}$ and (ii) $b_{1}=1.0 \mathrm{l} / \mathrm{mol}, b_{2}=3.0 \mathrm{l} / \mathrm{mol}, b_{3}=2.0 \mathrm{l} / \mathrm{mol}$. The remaining parameters are exactly the same as used in the above linear isotherm case. Plots of concentration profiles are shown in Figures 5 and 6. In both cases typical behavior of Langmuir isotherm can be observed. Once again, the reactant (components $\mathrm{C}$ ) is decreasing due to its conversion into two products (components A and B). In the current nonlinear case more reactant is converted into the products as compared to the linear case. Moreover, the conversion rate is much faster for the case having larger $b_{i}$ (for $i=1,2,3$ ). Figure 7 gives the plot of conversion with respect to the nonlinearity coefficients $b i$ which are taken same for all components, i.e. $b_{1}=b_{2}=b_{3}$.

It can be observed that conversion of the reactant increases on increasing the values of nonlinearity coefficients $b_{i}$. On the other hand, a comparison of Figures 2, 5 and 6 shows that separation of products is diminished on increasing the values of nonlinearity coefficients 
$b i$. The remaining observations are similar to those of linear case.

\section{Four-component reactive elution}

This section contains a case study of four-component reactive chromatography considering nonlinear reaction and either linear or nonlinear adsorption isotherms. Reactants are injected through inner cylindrical core of the inlet cross-section. All parameters used in this test problem are taken from Tien ${ }^{37}$ which have been considered in accordance with ranges typically encountered in HPLC applications. ${ }^{22}$

\section{Case 2a: Reaction of Methyl Formate with linear isotherm}

In this test problem, the reaction of type $A+B \leftrightarrows C+D$ is analyzed. The composition of reactants in the injected sample and flow-rate of the mobile phase are assumed to be constant. The general reaction mechanism is Methyl Formate+Water $\leftrightarrows$ Methanoic Acid+Methanol. In such a reaction, only component 1 (Methyl Formate) is injected as a reactant into a cylindrical column. The column is initially loaded only with component 2 (Water), while component 3 (Methanoic Acid) and component 4 (Methanol) are the reaction products. The parameters of reaction equilibrium, reaction kinetics, and adsorption isotherms are given in Table 4 for two different temperatures. The volumetric flow-rate is taken as $\dot{V}=0.1 \mathrm{ml} / \mathrm{min}$, $c_{1, \text { inj }}=0.5 \mathrm{~mol} / l, c_{2, \mathrm{inj}}=0=c_{3, \mathrm{inj}}=c_{4, \mathrm{inj}}$, velocity $u=6.216975 \mathrm{~cm} / \mathrm{min}$, porosity $\epsilon=0.24$, column length $L=25 \mathrm{~cm}$, column radius $R=1.25 \mathrm{~cm}$, radius of inner zone $\bar{r}=0.88375 \mathrm{~cm}$, mass transfer coefficient $k=50 \mathrm{~min}^{-1}$, dispersion coefficient $D_{z}=0.09925 \mathrm{~cm}^{2} / \mathrm{min}$ and $D_{r}=0.035 \mathrm{~cm}^{2} / \mathrm{min}$. The injected volume is $V=0.75 \mathrm{ml}$ and the time of injection is $t_{\text {inj }}=\frac{V}{V}=0.1333 \mathrm{~min}$. The chromatographic column is initially equilibrated with water only, i.e. $c_{2, \text { init }}=55.525 \mathrm{~mol} / l$ which is also injected to the column continuously, $c_{1, \text { init }}=0=$ $c_{3, \text { init }}=c_{4, \text { init }}$. The 2D-RLKM (c.f Eqs. (5) and (6)) is considered with linear equilibrium adsorption isotherms, $q_{i}^{*}=a_{i} c_{i}$ for $i=1,2,3,4$. The reaction rate of the heterogeneously 
catalyzed Methyl formate is described as

$$
r^{\text {het }}=k^{\text {het }}\left(q_{1} q_{2}-\frac{q_{3} q_{4}}{k_{\text {eq }}^{\text {het }}}\right)
$$

Here, the signs of the stoichiometric coefficients $\nu_{1}$ and $\nu_{2}$ are negative, while the signs of $\nu_{3}$ and $\nu_{4}$ are positive.

The eluted concentration profiles of component 1 (reactant) and reaction products (components 3 and 4) are shown in Figures 8 and 9. The reaction is considered at two different temperatures to further analyze the performance of the chromatographic reactors. The model parameters mainly depend on the temperature of the column besides porosity. The concentration profiles are generated using $100 \times 50$ mesh cells. The total elapsed time at temperature $T=298 K$ is 84.496 seconds and at temperature $T=318 K$ is 84.078 seconds. The plots of Figures 8 and 9 depicted that separation of the components 3 and 4 is significantly improved at high temperature. Moreover, conversion of the reactant into the product increases and the retention time reduces at higher temperature. For example, in Figure 9 a complete conversion of the reactant into products can be observed.

Figure 10 compares the experimental results of Tien $^{37}$ with our numerical simulations considering injection over the whole inlet cross-section of the column and large radial-dispersion coefficient. In this special case, the results of current 2D-model are equivalent to those of 1D-model. ${ }^{14}$ It can be observed that both numerical and experimental results are in good

agreement. However, we must point out that no experimental results are currently available characterizing 2D-isothermal reactive liquid chromatography.

\section{Case 2b: Reaction for Methyl Formate with nonlinear isotherm}

Here, we extend the previous case study to the nonlinear isotherm given by Eq. (3) for $i=1,2,3,4$. The Langmuir isotherm parameters are chosen as $b_{1}=1.0 \mathrm{l} / \mathrm{mol}, b_{2}=0 \mathrm{l} / \mathrm{mol}$, $b_{3}=1.0 \mathrm{l} / \mathrm{mol}$, and $b_{4}=1.0 \mathrm{l} / \mathrm{mol}$. The remaining data are the same as in the linear case. 
The numerical results are shown in Figures 11 and 12. Once again the reaction is considered at two different temperatures, i.e. $T=298 K$ and $T=318 K$. The elapsed time for the test problem is 85.182 seconds. Typical behavior of nonlinear Langmuir isotherms can be easily observed in the elution profiles. In the current four-component reaction, both conversion and separation are reducing on increasing the extent of nonlinearity.

\section{Conclusion}

This paper was based on the numerical approximation of a multi-component two-dimensional nonlinear and non-equilibrium model of isothermal reactive chromatography. The reaction rates were assumed to be nonlinear and adsorption isotherms were considered either linear or nonlinear. A semi-discrete HR-FVS was applied to solve the model equations numerically. Several practical case studies of three and four-component elution were considered and analyzed. It as found that the scheme avoids numerical oscillations and resolves the discontinuous profiles of the concentration profiles. It was further observed that high conversion can be achieved at high temperatures. Moreover, the retention time was significantly reduced at higher temperature. The current $2 \mathrm{D}$ model and numerical solutions illustrate the influence of mass transfer, longitudinal dispersion, and radial dispersion coefficients. The computed results could be very useful for understanding the transport mechanisms, to scale up physio-chemical parameters, and to optimize experimental conditions.

\section{Acknowledgement}

The first author is thankful to DAAD for financial support under funding program "Research Stays for University Academics and Scientists, 2016 (57210259)". 


\section{References}

(1) Carta, G. Simultaneous Reaction and Chromatography. In Chromatographic and Membrane Processes in Biotechnology; Costa, C. A., Cabral J. S., Eds.; Kluwer Academic Publishers: The Netherlands, 1991, p429.

(2) Schweich, D.; Villermaux, J. The Chromatographic Reactor. A New Theoretical Approach. Ind. Eng. Chem. Fundamen. 1978, 17, 1.

(3) Cho, B. K.; Aris, R.; Carr, R. W. The Mathematical Theory of a Countercurrent Catalytic Reactor. Proc. R. Soc. Lond. A 1982, 383, 147.

(4) Petroulas, T.; Aris, R.; Carr, R. W. Analysis of the Counter-Current Moving-Bed Chromatographic Reactor. Comput. Maths. Appl. 1985, 11, 5.

(5) Takeuchi, K.; Uraguchi, Y. Separation Conditions of the Reactant and the Product with a Chromatographic Moving Bed Reactor. J. Chem. Eng. Jpn. 1976, 9, 164.

(6) Takeuchi, K.; Uraguchi, Y. Basic Design of Chromatographic Moving Bed Reactors for Product Refining. J. Chem. Eng. Jpn. 1976, 9, 246.

(7) Takeuchi, K.; Uraguchi, Y. The Effect of the Exhausting Section on the Performance of a Chromatographic Moving Bed Reactor. J. Chem. Eng. Jpn. 1977, 10, 72.

(8) Takeuchi, K.; Uraguchi, Y. Experimental Studies of a Chromatographic Moving-Bed Reactor-Catalytic Oxidation of Carbon Monoxide on Activated Alumina as a Model Reactor. J. Chem. Eng. Jpn. 1977, 10, 455.

(9) Takeuchi, K.; Miyauchi, T.; Uraguchi, Y. Computational Studies of a Chromatographic Moving Bed Reactor for Consecutive and Reversible Reactions. J. Chem. Eng. Jpn. $1978,11,216$.

(10) Binous, H.; McCoy, B. J. Chromatographic Reactions of the Three Components: Application to Separations. Chem. Eng. Sci. 1992, 47, 4333. 
(11) Ganetsos, G.; Barker, P.E. Preparative and Production Scale Chromatography; Marcel Dekker, Inc.: New York, 1993; Vol. 61, p 375.

(12) Borren, T.; Fricke, J.; Schmidt-Traub, H. (Eds.). Chromatographic Reactors in Preparative Chromatography of Fine Chemicals and Pharmaceutical Agents; Wiley-VCH Verlag: Weinheim, 2005, p 371.

(13) Fricke, J.; Schmidt-Traub, H.; Kawase, M. Chromatographic Reactor, Ullmann's Encyclopedia of Industrial Chemistry; Wiley-VCH Verlag: Weinheim, 2005.

(14) Javeed, S.; Qamar, S.; Seidel-Morgenstern, A.; Warnecke, G. A Discontinuous Galerkin Method to Solve Chromatographic Models. J. Chromatogr. A 2011, 1218, 7137.

(15) Javeed, S.; Qamar, S.; Seidel-Morgenstern, A.; Warnecke, G. Parametric Study of Thermal Effects in Reactive Liquid Chromatography. Chem. Eng. J. 2012, 191, 426.

(16) Qamar, S.; Bibi, S.; Khan, F.U.; Shah, M.; Javeed, s.; Seidel-Morgenstern, A. Irreversible and Reversible Reactions in a Liquid Chromatographic Column: Analytical Solutions and Moment Analysis. Ind. eng. Chem. Res. 2014, 53, 2461.

(17) Lin, B.; Song, F.; Guiochon, G. A nalytical Solution of the Ideal, Nonlinear Model of Reaction Chromatography for a Reaction $\mathrm{A} \leftarrow \mathrm{B}$ and a Parabolic Isotherm. J. Chromatogr. A 2003, 1003, 91.

(18) Yamaoka, K.; Nakagawa, T. Moment Analysis for Reaction Chromatography. J. Chromatogr. A 1976, 117, 1.

(19) Sardin, M.; Schweich, D; Villermaux, J.; Ganetsos, G.; Barker, P.E. (Eds.). Preparative Fixed-Bed Chromatographic Reactor, Preparative and Production Scale Chromatography; Marcel Dekker Inc.: New York, USA, 1993, p 477.

(20) Villermaux, J.; Rodrigues, A.E.; Tondeur, D. (Eds.). The Chromatographic Reactor in 
Percolation Processes: Theory and application; Sijthoffen Noordhoff; Alpena an den Rijn: The Netherlands, 1981, p 539.

(21) Ruthven, D.M. Principles of Adsorption and Adsorption Processes; Wiley-Interscience: New York, 1984.

(22) Guiochon, G.; Felinger, A.; Shirazi, D.G., Katti, A.M. Fundamentals of Preparative and Nonlinear Chromatography, 2nd ed; ELsevier Academic press: New York, 2006.

(23) Javeed, S.; Qamar, S.; Seidel-Morgenstern, A.; Warnecke, G. Efficient and Accurate Numerical Simulation of Nonlinear Chromatographic Processes. J. Comput. Chem. Eng. 2011, 35, 2294.

(24) Leer, B.V. Towards Ultimate Conservative Finite Difference Scheme. IV. A New Approach to Numerical Convection. Scheme. J. Comput. Phys. 1977, 12, 276.

(25) LeVeque, R.J. Numerical Methods for Conservation Laws. Birkhaüser Verlag, Bassel, Germany, 1992.

(26) Cruz, P., Santos, J.C., Magalhães F.D.; Mendes, A. Simulation of Separation Processes Using Finite Volume Method.

J. Comput. \& Chem. Eng. 2005, 30,83.

(27) Lieres, E.V.; Andersson, J. A Fast and Accurate Solver for the General Rate Model of Column Liquid Chromatography. J. Comput. \& Chem. Eng. 2010, 34, 1180.

(28) Webley, P.A.; He, J. Fast Solution-Adaptive Finite Volume Method for PSA/VSA Cycle Simulation; 1 Single Step Simulation. J. Comput. \& Chem. Eng. 2000, 23, 1701.

(29) Koren, B. A Robust Upwind Discretization Method for Advection, Diffusion and Source Terms. In C. B. Vreugdenhil, B. Koren, editors, Numerical Methods for AdvectionDiffusion Problems, Volume 45 of Notes on Numerical Fluid Mechanics; Vieweg Verlag: Braunschweig, 1993, Chapter 5, p 117. 
(30) Parveen, S.; Qamar, S.; Seidel-Morgenstern, A. Analysis of Two-Dimensional NonEquilibrium Model of Linear Reactive Chromatography Considering Irreversible and Reversible Reactions. Ind. Eng. Chem. Res. 2016, 55, 2471.

(31) Bibi, S.; Qamar, S.; Seidel-Morgenstern, A. Irreversible and Reversible Reactive Chromatography: Analytical Solutions and Moment Analysis for Rectangular Pulse Injections. J. Chromatogr. A 2015, 1385, 49.

(32) Thiele, A.; Falk, T.; Tobiska, L.; Seidel-Morgenstern, A. Prediction of Elution Profiles in Annular Chromatography. Chem. Eng. Sci. 2001, 25, 1089.

(33) Danckwerts, P.V. Continuous Flow Systems. J. Chem. Eng. Sci. 1953, 2, 1.

(34) Parveen, S.; Qamar, S.; Seidel-Morgenstern, A. Two-Dimensional Non-Equilibrium Model of Liquid Chromatography: Analytical Solutions and Moment Analysis. Chem. Eng. Sci. 2015, 122, 64.

(35) Roe, P.L. Characteristic-Based Schemes for the Euler Equations. Annual Review of Fluid Mechanics 1986, 18, 337.

(36) Gottlieb, S.; Shu, C.-W. Total Variation Diminishing Runge-Kutta Schemes, Math. Comput. 1998, 67, 73.

(37) Tien, V.D. Analysis of Heterogeneously Catalyzed Ester Hydrolysis Reactions in a FixedBed Chromatographic Reactor, PhD thesis; Faculty of Process and System Engineering, OVGU, Magdeburg, Germany, 2007.

Table 1: Different flux limiters used in Eq. (23)

\begin{tabular}{|c|c|}
\hline Flux limiter & Formula \\
\hline van Leer $\left(^{24}\right)$ & $\varphi(r)=\frac{|r|+r}{1+|r|}$ \\
Superbee $\left({ }^{35}\right)$ & $\varphi(r)=\max (0, \min (2 r, 1), \min (r, 2))$ \\
Minmod $\left(^{35}\right)$ & $\varphi(r)=\max (0, \min (1, r))$ \\
MC $\left({ }^{24}\right)$ & $\varphi(r)=\max \left(0, \min \left(2 r, \frac{1}{2}(1+r), 2\right)\right)$ \\
\hline
\end{tabular}


Table 2: Single-component non-reactive elution: $L^{1}$-error and CPU times of schemes at $100 \times 50$ grid points.

\begin{tabular}{|c|cccc|c|}
\hline Limiter & \multicolumn{3}{|c|}{$L^{1}$-error } & CPU $(\mathrm{s})$ \\
\hline & $D_{\mathrm{z}}=10^{-3}$ & $D_{\mathrm{z}}=10^{-4}$ & $D_{\mathrm{z}}=10^{-5}$ & $D_{\mathrm{z}}=10^{-6}$ & $D_{\mathrm{z}}=10^{-3}$ \\
\hline First order & 0.0504 & 0.0630 & 0.0744 & 0.0624 & 9 \\
Koren & 0.0009 & 0.0038 & 0.0054 & 0.0055 & 14 \\
van Leer & 0.0037 & 0.0065 & 0.0076 & 0.0077 & 15 \\
Superbee & 0.0043 & 0.0047 & 0.0055 & 0.0056 & 16 \\
Minmod & 0.0071 & 0.0207 & 0.0206 & 0.0207 & 19 \\
MC & 0.0032 & 0.0031 & 0.0039 & 0.0061 & 15 \\
\hline
\end{tabular}

Table 3: Single-component non-reactive elution: $L^{1}$-error and EOC of the Koren scheme.

\begin{tabular}{|c|ll|ll|ll|}
\hline Grid points & $D_{z}=10^{-3}$ & & $D_{z}=10^{-4}$ & & $D_{z}=10^{-5}$ & \\
\hline$N_{x} \times N_{\rho}$ & $L^{1}$-error & EOC & $L^{1}$-error & EOC & $L^{1}$-error & EOC \\
\hline $20 \times 50$ & $4.6 \times 10^{-3}$ & & $8.4 \times 10^{-3}$ & & 0.012 & \\
$40 \times 50$ & $1.5 \times 10^{-3}$ & 1.6 & $2.5 \times 10^{-3}$ & 1.7 & $3.7 \times 10^{-3}$ & 1.7 \\
$80 \times 50$ & $4.9 \times 10^{-4}$ & 1.6 & $6.2 \times 10^{-4}$ & 2.0 & $8.6 \times 10^{-4}$ & 2.1 \\
$160 \times 50$ & $1.5 \times 10^{-4}$ & 1.7 & $1.6 \times 10^{-4}$ & 2.0 & $1.9 \times 10^{-4}$ & 2.2 \\
$320 \times 50$ & $4.4 \times 10^{-5}$ & 1.8 & $4.3 \times 10^{-5}$ & 1.9 & $3.8 \times 10^{-5}$ & 2.3 \\
\hline
\end{tabular}

Table 4: Adsorption and reaction coefficients for Methyl Formate. ${ }^{37}$

\begin{tabular}{|c|c|c|c|c|c|c|}
\hline Temperature & \multicolumn{4}{|c|}{$a_{i}$} & $k^{\text {het }}$ & $k_{\text {eq }}^{\text {het }}$ \\
\hline & 1 & 2 & 3 & 4 & $\min ^{-1}$ & \\
\hline $298 K$ & 1.110 & 1.0 & 0.723 & 0.458 & $5.88 \times 10^{-3}$ & 0.1134 \\
\hline $318 \mathrm{~K}$ & 1.038 & 1.0 & 0.768 & 0.445 & $41.6 \times 10^{-3}$ & 0.1449 \\
\hline
\end{tabular}

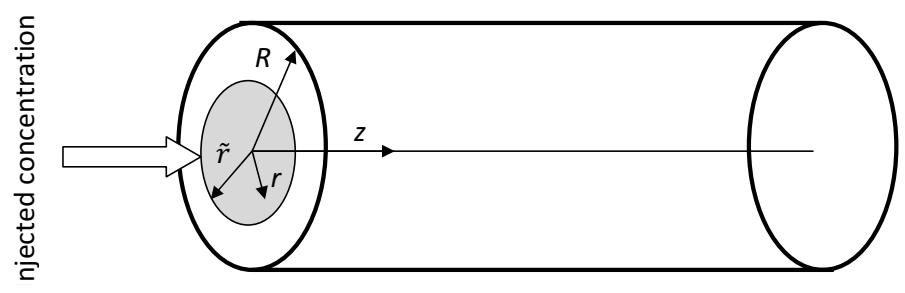

Figure 1: Process diagram of a chromatographic reactor of cylindrical geometry. 

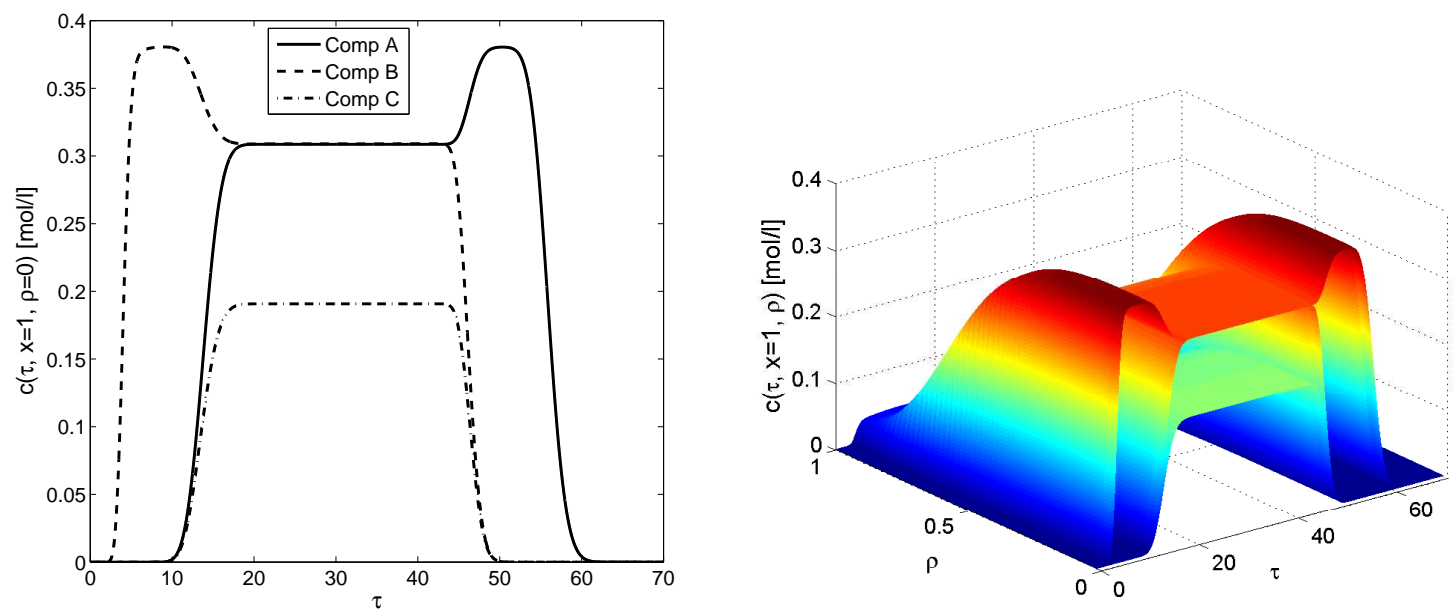

Figure 2: Case 1a: Three-component reactive elution profiles with linear isotherm.

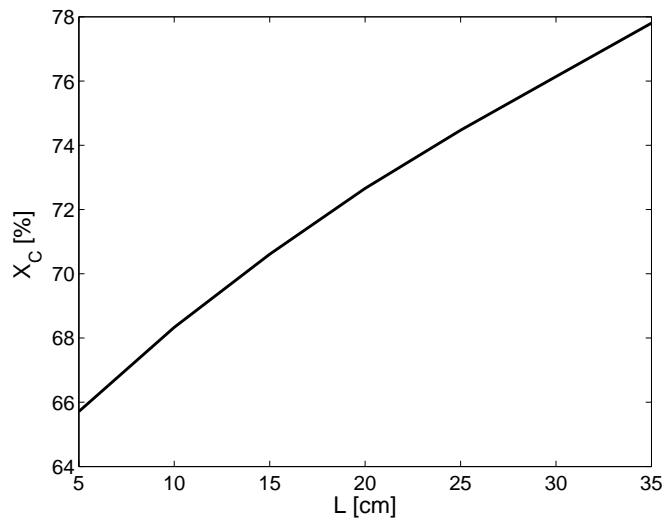

Figure 3: Case 1a: Plot of reactant conversion with respect to column length using linear isotherm. 
(a)

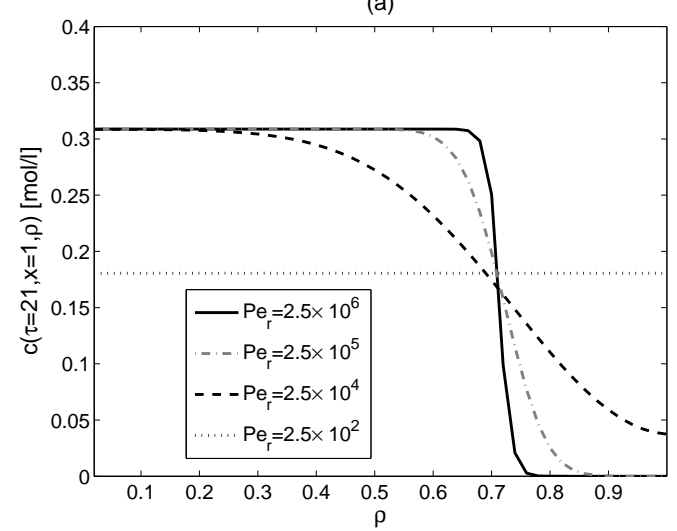

(b)

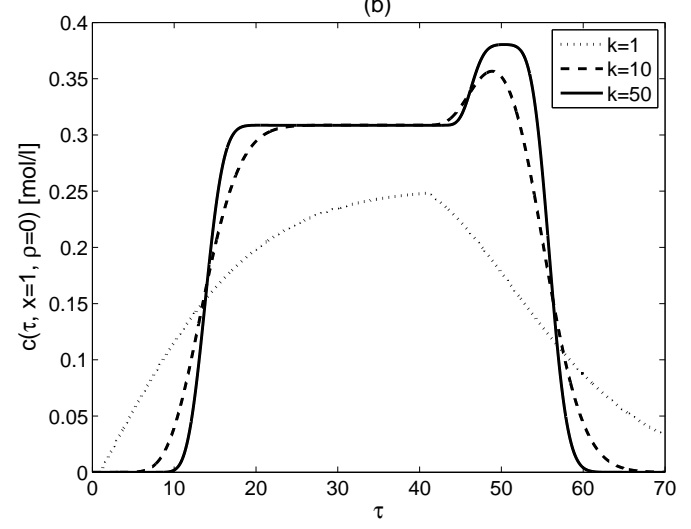

Figure 4: Case 1a: Effects of $P e_{r}$ and mass transfer coefficient $k$ on component 1 in threecomponent reactive elution using linear isotherm.
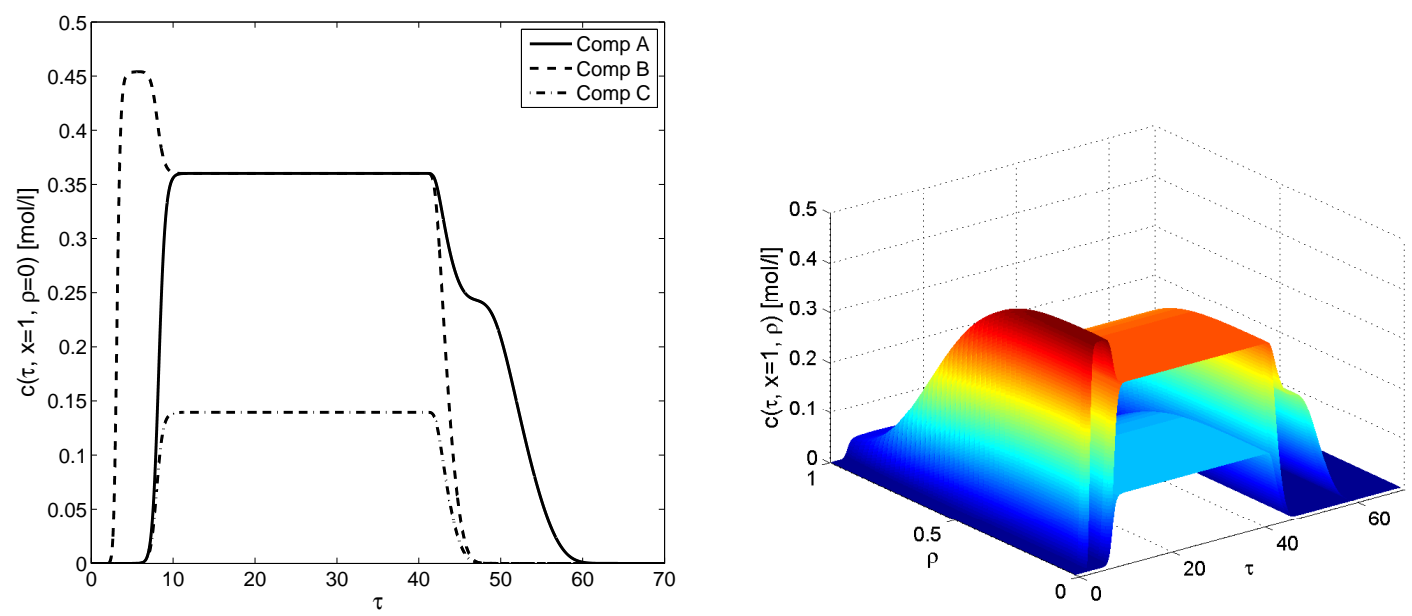

Figure 5: Case 1b: Three-component reactive elution profiles using nonlinear isotherm. Here, $b_{A}=1.0 \mathrm{l} / \mathrm{mol}, b_{B}=1.0 \mathrm{l} / \mathrm{mol}$, and $b_{C}=1.0 \mathrm{l} / \mathrm{mol}$. 

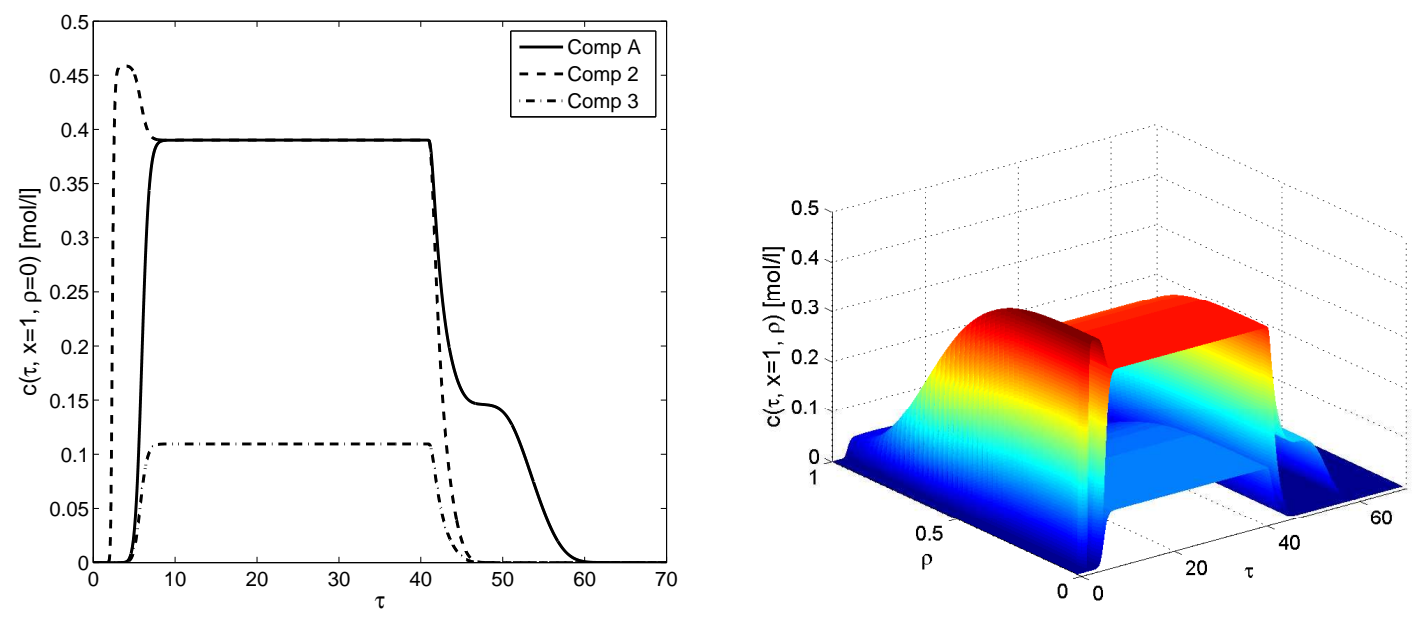

Figure 6: Case 1b: Three-component reactive elution profiles using nonlinear isotherm. Here, $b_{A}=1.0 \mathrm{l} / \mathrm{mol}, b_{B}=3.0 \mathrm{l} / \mathrm{mol}$, and $b_{C}=2.0 \mathrm{l} / \mathrm{mol}$.

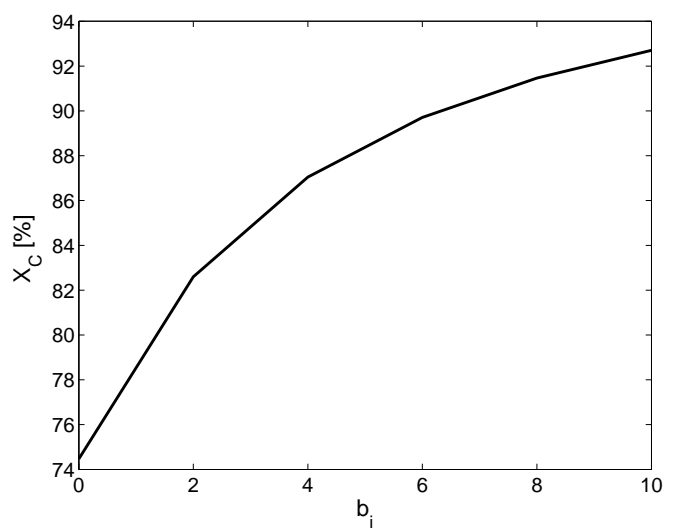

Figure 7: Case 1b: Plot of reactant conversion for various $b_{i}$ in three-component reactive elution. Here, we have chosen $b_{A}=b_{B}=b_{C}$. 

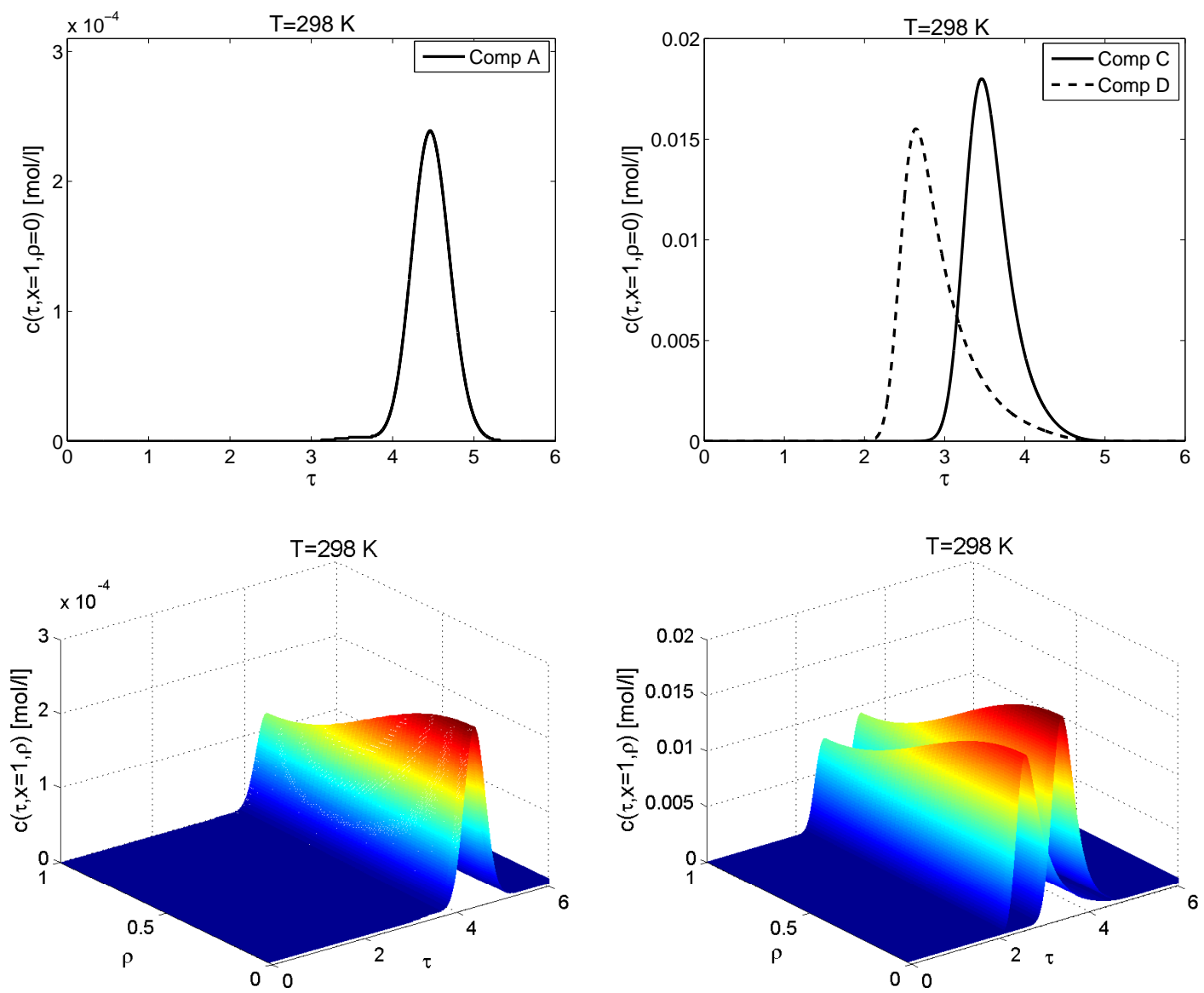

Figure 8: Case 2a: Four-component reaction: Elution profiles with linear isotherm at $T=$ $298 K$. 

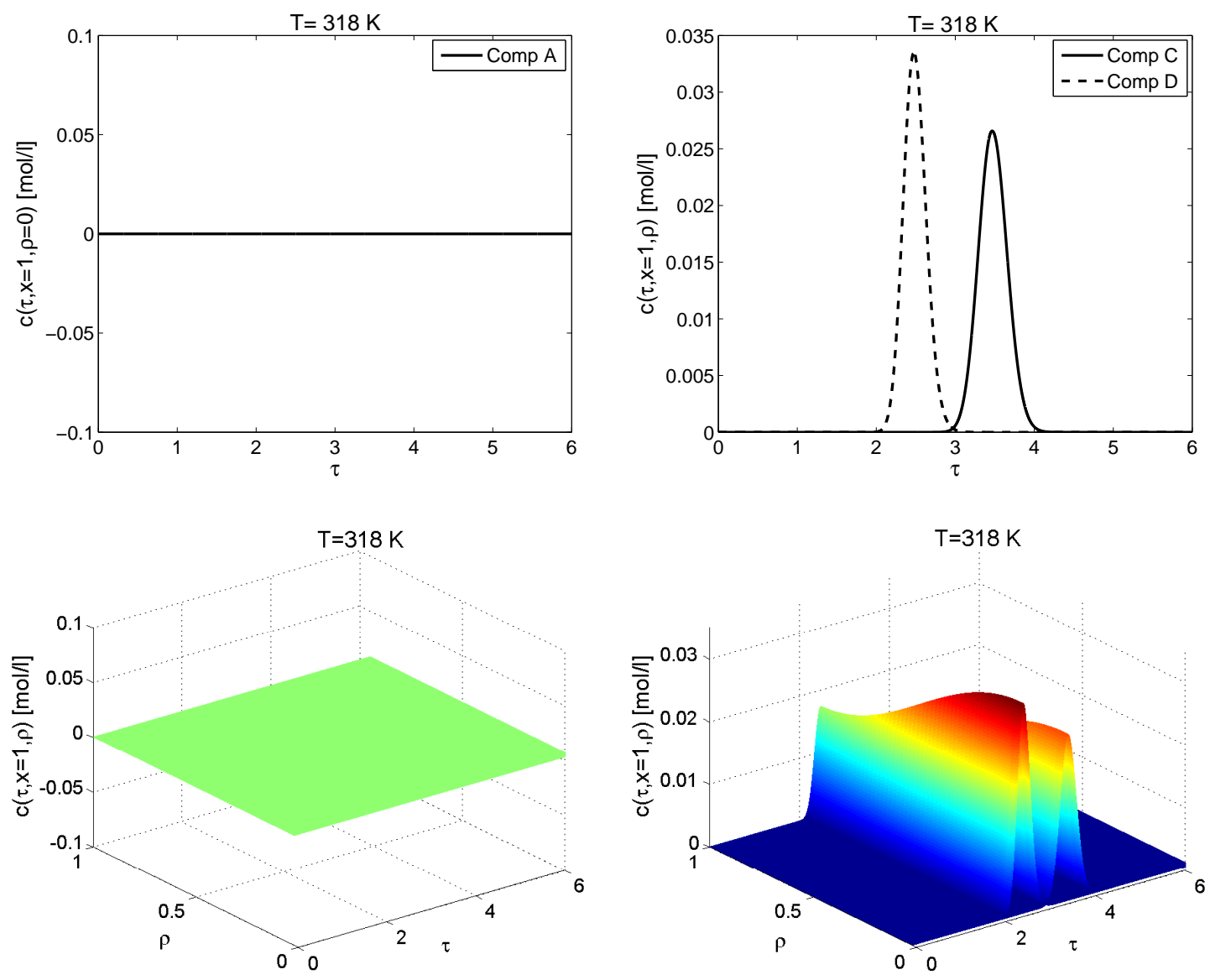

Figure 9: Case 2a: Four-component reaction: Elution profiles with linear isotherm at $T=$ $318 K$.

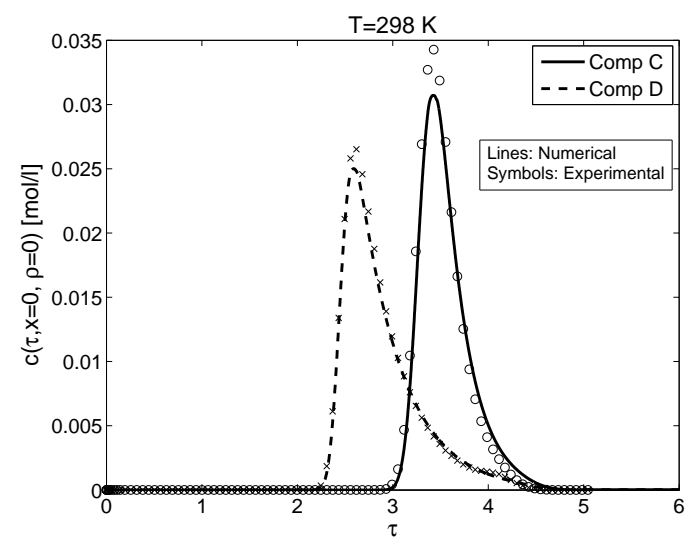

Figure 10: Case 2a: Four-component reaction: Comparison of numerical and experimental results at $T=298 \mathrm{~K}$. Here, lines represent numerical solutions and symbols denote experimental solutions. 

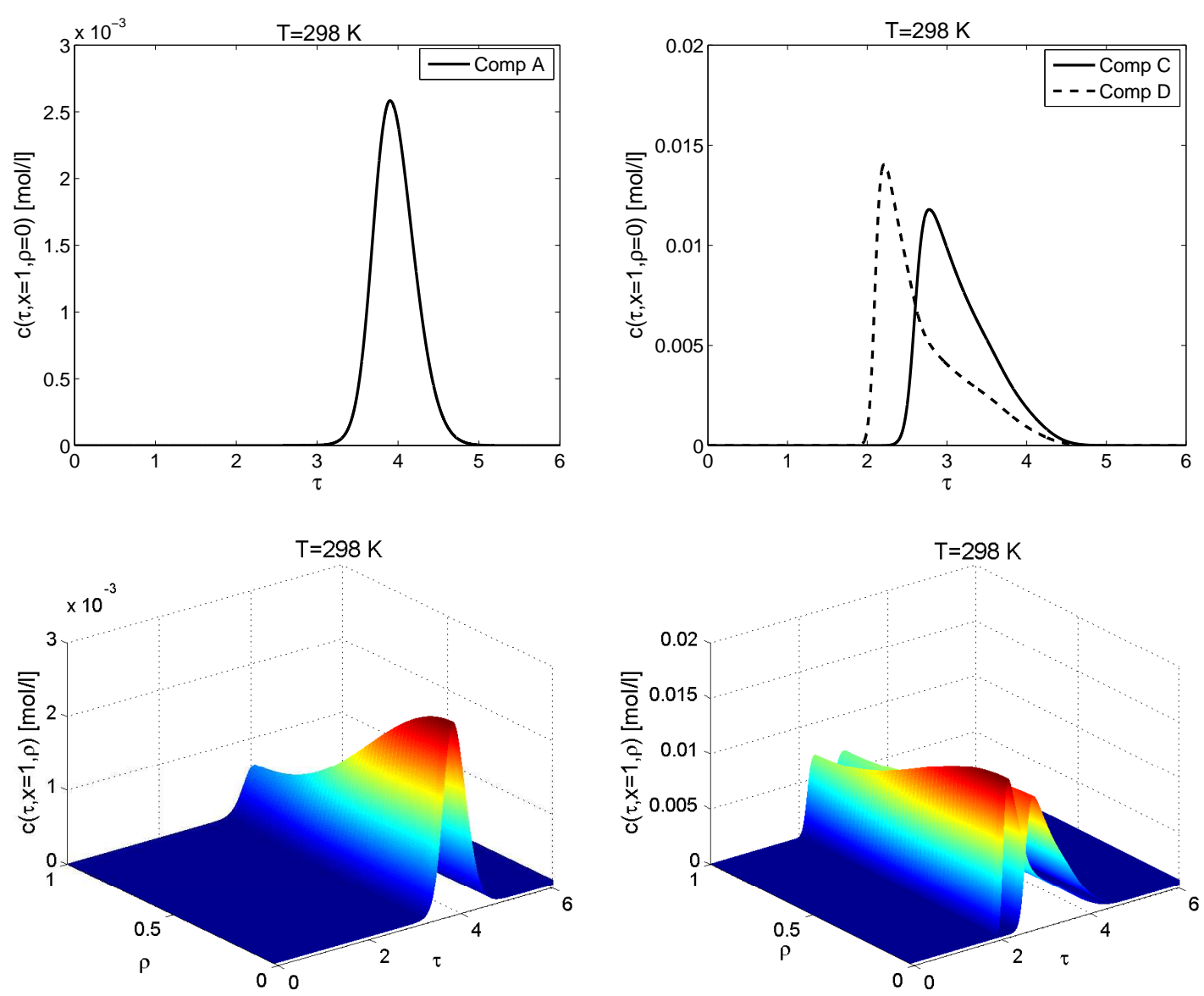

Figure 11: Case 2b: Four-component reaction: Elution profiles with nonlinear isotherm at at $T=298 K$. 

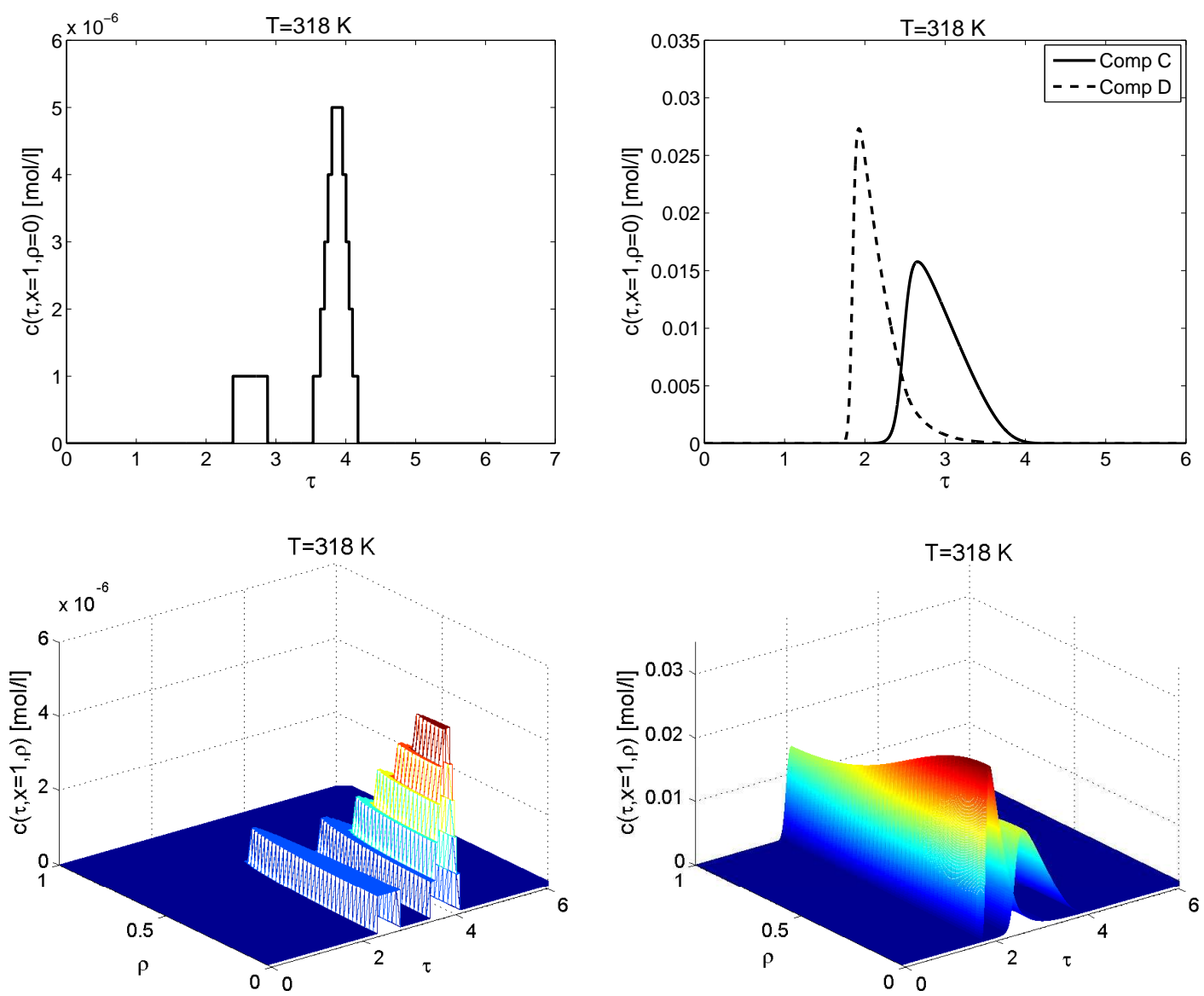

Figure 12: Case 2b: Four-component reaction: Elution profiles with nonlinear isotherm at $T=318 K$.

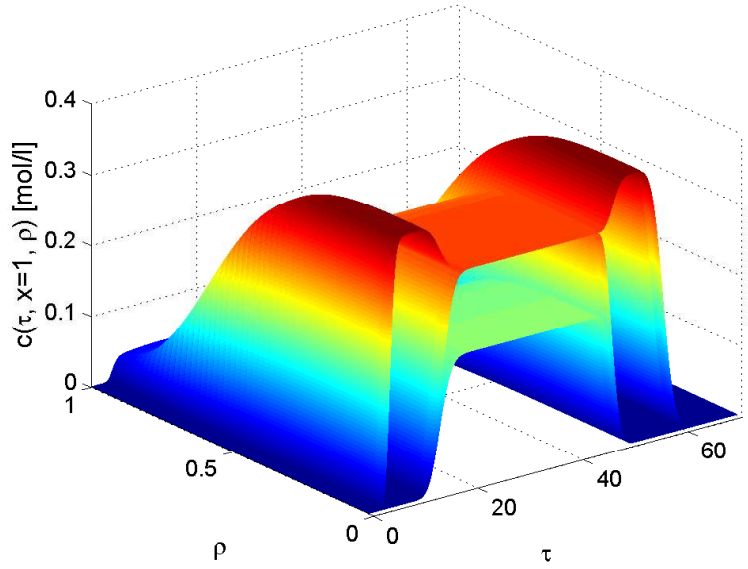

For Table of Contents only. 